\title{
Mapping Attitude Formation as a Function of Information Input: Online Processing Models of Attitude Formation
}

\author{
Jacob Jacoby \\ Department of Marketing \\ New York University \\ Maureen Morrin \\ Department of Marketing \\ Boston University \\ James Jaccard \\ Department of Psychology \\ State University of New York-Albany \\ Zeynep Gurhan \\ Department of Marketing \\ University of Michigan \\ Alfred Kuss \\ Department of Marketing \\ Fern Universitat, Hagen, Germany \\ Durairaj Maheswaran \\ Department of Marketing \\ New York University
}

\begin{abstract}
Consistent with calls for a dynamical social psychology (Jacoby et al., 1987; Nowak, Lewenstein, \& Vallacher, 1994), attitude formation was studied using a recently developed, computer-based simulation technique termed Higher Order Cognitive Tracing (see Jacoby et al., 1994). Participants' attitudes toward 12 different products in 3 product categories were investigated as a function of incremental information input. As opposed to traditional memory-based models of attitude formation, the study explored online processing models. Results indicate that the impact of information tends to decrease the later in the sequence that information is accessed. In addition, new information that is affectively inconsistent with prior information tends to have a greater impact on attitudes than information that is affectively redundant. This effect is more pronounced earlier rather than later in the sequence of information acquisition. Implications and suggestions for future research are discussed.
\end{abstract}

Noted psychologists have recently called attention to several interrelated problems with social psychological research.

Maureen Morrin is now at the University of Pittsburg. Alfred Kuss is now at the Frei Universitat, Berlin, Germany.

Requests for reprints should be sent to Jacob Jacoby, Department of Marketing, Stern School of Business, 44 West 4th Street, New York, New York10012. E-mail: jjacoby@stern.nyu.edu
Identifying the need to pay greater attention to issues of development and change, Deaux wrote the following:

Social psychologists always take the cross-sectional cut in their research. These designs yield information about how processes operate at one particular point in time, but tell us little about where they come from or where they are going. In commenting on this disciplinary emphasis, Costanzo (1991) recently lik- 
ened social psychology to Chapter 4 of an eight-chapter book. That chapter is very thorough; but how about paying a little attention to chapters 1 through 3 and 5 through 8 , he asked. (1993, pp. 10-11)

With specific respect to the attitude construct, Eagly (1992) encouraged attitude researchers to go beyond their current research paradigms because these "allow their theories to be seriously limited by the constraints of their research paradigms, which often allow only certain processes to be manifest. Theory encapsulated within an experimental paradigm is thus limited in scope" (p. 704). This echoes the long-standing scientific understanding that methods shape and limit theory (e.g., Kaplan, 1963; Kuhn, 1970), a notion that applies equally well to social psychology (e.g., Gergen, 1973; McGuire, 1973; Rosnow, 1981; Vallacher \& Nowak, 1994). This article introduces a relatively new methodological tool, Higher Order Cognitive Tracing (HOCT; see Jacoby et al., 1994), that can be used to study the dynamic emergence of cognitions and attitudes in the context of information processing. We illustrate the application of the method to the analysis of attitude formation in the context of online processing of information.

\section{ATTITUDE DEVELOPMENT AND CHANGE}

As noted by Eagly \& Chaiken $(1984,1993)$, attitudes follow from different types of input. One such input is information acquired either from the external environment or personal experience. Many approaches focus on the informational bases of attitudes. For example, in characterizing the expectancy-value approach to attitudes, Fishbein and Ajzen (1975) wrote the following: "It is clear that this approach postulates an informational basis for the formation of attitude; a person is viewed as processing the information he has about an object in arriving at his evaluation of the object" (p. 222). Others noting the critical nature of informational input for attitude formation include Anderson (1971) and Jaccard and Wood (1986; see also Jaccard, Wan, \& Wood, 1988).

In commenting on the persuasion paradigm typically used for studying the effects of information on attitude development and change, Eagly (1992) observed the following: "The information that is presented generally consists of relatively complex verbal messages whose components are an overall position that is advocated and one or more arguments designed to support that position" (p. 699). Information impact is typically assessed via preexposure versus postexposure designs, where exposure means all-at-once exposure to the entire "relatively complex verbal message." As a consequence, one can determine that a change has occurred, but unless one systematically varies the information elements across different experimental groups, one cannot be able to determine which element exerted what impact. Not only does this approach become unwieldily when messages consist of many elements (as is generally the case with most "relatively com- plex verbal messages"), at best, it enables us only to explore what happens at the aggregate level, not the individual level. Needed are empirical procedures that permit examination (preferably at the individual level) of how molecular changes in information acquisition are directly related, in one-to-one fashion, to molecular changes in attitude development and change, phenomena theorized to change as a function of information input.

Although theorists have speculated regarding the nature of attitude development as a function of incremental information input (e.g., Fishbein \& Ajzen, 1975, p. 223), such speculations either remain untested or have been tested via mathematical weighted averaging models applied to aggregates (see Anderson, 1971, 1981, 1991; Himmelfarb, 1973; Sloan \& Ostrom, 1974). Insofar as can be determined, most likely due to the absence of an appropriate methodology, few studies have been reported that examine the impact of item-by-item information acquisition on attitude formation. Research does exist that has examined serial processing of information on a trial-by-trial basis (e.g., Anderson, 1981; Schlottmann \& Anderson, 1995), but this work has not focused on attitudinal judgments. The development and application of an appropriate methodology for studying item-by-item processing would be consistent with calls for a dynamic conception of attitude consistency and change (Eiser, 1994). "Indeed, it is only ky tracking the evolution of a systems behavior on some time scale that the essence of a complex system can be identified" (Vallacher \& Nowak, 1994, p. 9).

\section{MEMORY-BASED VERSUS ONLINE PROCESSING OF INFORMATION IN THE FORMATION OF ATTITUDES}

Hastie and Park (1986) distinguished two types of information processing models for the formation of social judgments: (a) memory-based models, and (b) online processing models. In the context of attitude formation, memory-based models are those where an individual recalls from memory information about an attitude object and then, based on that information, forms an attitude toward the object. Memory-based models emphasize the process of accessing from memory information that was acquired on a previous occasion (although that occasion may have occurred only minutes ago), with the assumption being that the attitude is some function of the information that is retrieved from memory (e.g., salient beliefs in Fishbein's expectancy-value approach). Online processing models, by contrast, emphasize a process whereby an attitude is formed after the acquisition of an initial piece of information and then revised and updated online as each subsequent piece of information is acquired. The final attitude is then stored in memory and occupies a separate memory bin than the information on which it was based. Indeed, the original information may be forgotten or inaccessible, but the attitude on which it is based remains in memory and available for recall. 
The vast majority of research in the attitude formation area has focused on memory-based models of the attitude formation process or has used methodologies that ignore the sequential character of online processing. For example, in Fishbein's expectancy-value formulation, the most common application is to elicit from an individual his or her salient beliefs and then to assess expectancy and value components for each belief. These component measures are combined in accord with expectancy-value theory and then correlated with an independent measure of attitude. This approach clearly emphasizes memory-based orientations to the analysis of the relation between information (beliefs) and attitudes. Anderson's (1981, 1991) information integration theory is typically applied by presenting individuals with descriptions of hypothetical attitude objects using multiple pieces of information per description. The individual's attitude toward each object is measured after exposure to the information and the attitude ratings are used to infer the scale values of the information used to describe a given object and the integration rule that was used to combine the individual pieces into an overall global attitudinal judgment. Such applications measure a single attitude after full exposure to all information and thus, are not amenable to the analysis of the emergence of attitudes in an online processing sense. This is also true for attitudinal analyses using conjoint measurement as a theoretical framework.

A general model of attitude formation for memory-based processing can be stated using Anderson's information integration theory as a general framework:

$$
A t t=w_{0} A t t_{0}+w_{1} s_{1}+w_{2} s_{2}+\ldots+w_{k} s_{k}
$$

where Att is the attitude in question, Atto is the previously stored attitude in memory, if any, $s$ is the scale value for a given piece of information recalled from memory, $w$ is a weighting parameter reflecting the importance of the information in determining the attitude, and $\mathrm{k}$ is the number of pieces of information recalled from memory. By contrast, a model of online processing can be specified as follows:

$$
A t_{t}=w_{1} A t_{t-1}+w_{2} s_{t}
$$

where $\mathrm{Att}_{\mathrm{t}}$ is the attitude toward the object at time $t, \mathrm{Att}_{\mathrm{t}-\mathrm{l}}$ is the attitude toward the object prior to the acquisition of information at time $t, s_{t}$ is the scale value of the piece of information acquired at time $t$, and $w_{1}$ and $w_{2}$ are weighting parameters that reflect the importance of the prior attitude and current piece of information in determining the current attitude. Online processing minimizes information storage demands in memory and also minimizes the number of pieces of information that must be processed at a given time (because there are only two pieces of information, the previous attitude and the current information). Because of this, online processing is often viewed as an efficient (but not necessarily optimal) serial processing strategy (Anderson, 1981, 1991; Busemeyer, 1991; Hogarth \& Einhorn, 1992; Schlottmann \& Anderson, 1995). It seems obvious that a large number of decisions and judgments derive from online processing of information
(Hogarth \& Einhorn, 1992). Despite this, there has been a paucity of research about how attitudes (as defined by overall judgments of positive or negative affect toward an object) form in such contexts. This article describes a methodology that can be used for studying such processing and applies the framework to the analysis of information impact on the formation of attitudes and explores several theoretical issues (described later) for models of online attitude formation.

\section{HIGHER ORDER COGNITIVE TRACING}

Whether implicitly or explicitly, virtually all attitude theorists subscribe to some form of an Hierarchy of Effects Model whereby incoming information (e.g., a message transmitted by a source) reaching the receiver is presumed to result in a sequence of activities going from exposure, through attention, comprehension, belief, evaluation, intention formation, retention, and ultimately to overt behavior (e.g., McGuire, 1976). A key assumption is that the earlier stages exert a limiting influence on subsequent "higher order" stages (see especially, McGuire, 1972). For example, if information has not been acquired, then it can have no direct impact on belief or attitude formation. Thus, an important question becomes "What impact do incremental changes in earlier stages have on the development and change of later stages?"

Rather than being a static or all-at-once phenomenon, each of the hierarchy's stages is presumed to be a dynamic process in its own right. Information acquisition is no exception (see Jacoby et al., 1987). In this context, interest attaches to information acquisition because of its presumed impact on the formation and change of attitudes. As alluded to by Costanzo (1991), Deaux (1993), Eagly (1992), Eiser (1994), Jacoby $(1975,1977)$, and Vallacher and Nowak (1994), among others, to better capture and study higher order phenomena theorized to operate as processes, one needs a methodology that is in greater correspondence with, and permits more adequate examination of, process formulations. Process methods are specifically needed for capturing the development of attitudes in a way that links them directly, in one-to-one fashion to item-by-item information acquisition. HOCT is such a procedure. An extension of existing Behavioral Process procedures for studying the dynamic nature of information acquisition, HOCT was devised for studying the impact of item-by-item information acquisition on the development and change of higher order mental processes.

As described in Jacoby et al. $(1977,1987)$, the behavioral process approach is a simulation strategy that involves the following: First, the individual is confronted with either a choice or evaluation task. Second, that person is provided with access to an environment containing information that may be authentic, hypothetical, or some combination of the two. This information environment may be conceptualized as a two-dimensional "options $\times$ properties" plane or as a three-dimensional "options $\times$ properties $\times$ sources" cube. Options represent objects, people, organizations, or alternative courses of action. 
As per Torgerson (1958), properties are describable characteristics of options (e.g., different brands of a product generally vary in terms of their price, name, ingredients, manufacturer, image, etc.). Sources represent entities from which property $x$ option information can be obtained. Third, the problem solver is permitted to acquire information, while a trace of this information accessing behavior is captured and preserved. In addition to learning the outcome of the process (e.g., which of several options was selected or the final evaluation of the options), the data indicate "how much" and "just which" information went into arriving at the outcome, and "in what order" this occurred. Such data have been referred to as the depth, content, and sequence of information accessing, respectively.

In concept, the HOCT extension is simple. It involves introducing an additional "side-step" into the information accessing process such that, immediately after accessing one item of information, but before accessing another, the individual is required to respond to a scale that taps into a higher order mental process (e.g., an attitude). Only after that side-step is completed can the person access more information. Assuming that the side-step is not reactive (in the sense of affecting subsequent information accessing or the dependent variable itself), this permits examination of the molecular changes in the higher order process as a function of item-by-item information acquisition. Although it makes no assumptions regarding the comprehension or miscomprehension of information, by its very nature, HOCT identifies just which of the available information has at least been accessed by the individual.

Among the myriad of cognitive processes that can be studied in the context of attitude and judgment-based research, the HOCT procedure is best suited to addressing questions focused on how judgments unfold as each piece of new information is acquired. Applications of the procedure must consider the possibility that the task is reactive in that it forces individuals to update their judgment after each piece of information is acquired. Current research with the technique has explored the issue of reactivity across several tasks by comparing judgments that people make at various points in the acquisition sequence when they are using HOCT as opposed to the more traditional paradigm that does not require a judgment after each piece of information (Jacoby et al., 1994). In general, there is little evidence for reactivity. Issues of external validity are also relevant, namely that of how often and in what contexts people process information and form judgments in a sequential updating fashion. There is no research that we are aware of that has addressed this issue. The general strategy of measuring attitudes prior to a message and then again after the message has been processed ignores the possibility that although we read stories, examine ads, read the information on a package, or watch a television commercial, we are having affective reactions or forming attitudes as we go. The working assumption is that such phenomena are of little consequence or that people suspend judgments until all of the information is acquired. This seems unrealistic. HOCT provides one approach to examining how judgments, affective reactions, or other such phenomena unfold as information is acquired.

Other methods have been used to capture how people react dynamically to information. For example, persuasion research based on the elaboration likelihood model (Petty \& Cacioppo, 1981) asks people to recall the thoughts they had while they were reading or viewing a persuasive message. It is not our purpose to contrast the HOCT strategy with these other approaches, such as verbal protocols, think aloud strategies, analysis of eye movements, and recalled thought, as the relevant dimensions along which many of these methods differ are discussed elsewhere (c.f., Jacoby, Jaccard, Kuss, Troutman, \& Mazursky, 1987). Rather, our purpose is to introduce researchers to this relatively new empirical strategy that represents an additional methodological tool for the analysis of process-based phenomena.

\section{ANALYSES OF ONLINE ATTITUDE FORMATION}

In this article, we illustrate the application of the HOCT procedure to the analysis of online attitude formation. Specifically, we analyze the unfolding of attitudes as affectively redundant information about an attitude object is acquired and we also explore the impact of the introduction of affectively inconsistent information.

There has been considerable research on the impact of affectively redundant information on attitude formation. Most of this research has evolved from the controversy on summation versus averaging integration rules (e.g., Anderson, 1981), in which affectively redundant information has been shown to either raise, lower, or have no impact on an existing attitude, depending on the type of integration rule operating and whether initial attitudes were included in the model (see Anderson, 1981). For the online processing model in Equation 2, a reasonable hypothesis is that the absolute weight attached to the preexisting attitude $\left(\mathrm{w}_{\mathrm{t}-1}\right)$ increases as information accumulates and the cognitive base from which the attitude is derived becomes more numerous and diverse. This proposition is consistent with recent research on attitude strength (Jaccard, Radecki, Wilson, \& Dittus, 1995; Petty \& Krosnik, 1995), which suggests that attitudes are stronger and more resistant to change as the number of beliefs underlying the attitude increases. Assuming a constant scale value for each new piece of information (i.e., affectively redundant information), this proposition implies that the impact of information on the attitude will decrease with each successive piece of information that is acquired, yielding the following formal hypothesis: The later an item of information is considered, the lower is its impact on attitude formation. A large body of research has explored order effects in judgment tasks and a typical result is that of primacy, just as we predict. The classic paradigm presents two groups of individuals with identical information about an object (e.g., a person or a prod- 
uct), half of which is positive in character and half of which is negative. One group receives the positive information first followed by the negative information, whereas the other group receives the negative information first followed by the positive information. It is typically found that individuals in the Pro-Con condition develop more favorable attitudes toward the object than people in the Con-Pro condition, suggesting that the initial information had a larger impact than the later information. The exact mechanism underlying this result is unclear, although theorists have suggested such phenomena as greater attention to early information, discounting of later (affectively inconsistent) information, as well as a change-in-meaning phenomena where the initial information alters the meaning of the later information (Anderson, 1981; Jaccard \& Fishbein, 1975). Research has been unable to disentangle these mechanisms and it is possible that all operate simultaneously to some extent. An online processing model suggests yet an additional mechanism that may account for primacy effects, namely that as more information is accrued, the prior attitude at time $t-1$ carries increasingly greater weight. Later information has less impact because by the time that such information is acquired, the prior attitude is given large weight in the integration process. The traditional experimental design for assessing order effects in attitude judgments is problematic because it provides no perspectives on how judgments change as each piece of information is acquired. One knows that earlier information must have had a greater impact overall, but exactly how the attitude unfolds is unclear. Also, the traditional paradigm cannot provide perspectives on the nature of order effects when all of the information is of the same affective sign. Indeed, order effects can only be analyzed at the group level, not on an individual-by-individual basis. HOCT provides a methodology that allows one to assess the effects of sequencing of information as each piece of information is acquired and it does so at both the individual and group level.

In this study, we trace how the attitude toward an object changes with each additional piece of information. We present information curves which plot attitude change induced by a piece of information as a function of the order in which the information is accessed. Separate information curves are formed for the case where the information is almost all positive, almost all negative, and where there is a mixture of positive and negative information. There is considerable literature to suggest that people tend to exhibit a negativity bias, whereby they attend to and give greater weight to negative as opposed to positive information (Jaccard \& Becker, 1985). This suggests that the information curves may have different forms depending on whether the information is primarily positive or negative in character. For example, one might expect that additional negative information will continue to exert an impact longer than will be the case for additional positive information. The information curves provide perspectives on this possibility. A second issue to be addressed focuses on the impact of affectively novel infor- mation on online attitude formation. As noted previously, theorists have suggested that information that is affectively inconsistent with the initial information is subject to discounting on the part of the individual. In this research, we utilized the HOCT procedure to examine how attitudes unfold in the context of online processing and how attitudes change when affectively inconsistent information is acquired relative to information that has been acquired previously. The research is distinct from previous analyses because it does not rely on the comparison of overall attitudes between experimental conditions where the nature and flow of information is strictly controlled and varied. Rather, the changes in attitude are traced with the acquisition of each new piece of information, so that the impact of a given instance of affectively inconsistent information can be identified. This permits more fine-grained analyses of the information acquisition process and its impact on attitudes. The discounting hypotheses holds that information should have less of an impact when it is preceded by information that is affectively inconsistent with it on previous trials. This is due to a tendency to discount the inconsistent information or to assimilate it toward the already-formed attitude. However, an alternative theoretical account based on novelty and contrast effects makes an opposing prediction. According to this view, when a piece of information is preceded by information that is affectively opposite to it, it makes the current piece of information appear novel and to stand out relative to the prior information. This novel information may, in turn, have an increased impact on attitude by virtue of its increased salience. Another purpose of this study was to evaluate these opposing predictions. In sum, social psychological analyses of attitude have been dominated by memory-based models as opposed to online models and have tended to use methodological procedures that ignore the dynamic nature of attitudes as information is acquired in succession. This study illustrates the use of an innovative methodological paradigm for studying the unfolding nature of judgment phenomena, identifies and analyzes information curves that show the impact of each successive piece of information, and tests competing predictions of a discounting versus novelty conceptualization of the effects of affectively inconsistent information.

\section{METHOD}

\section{Sample}

The sample consisted of $\mathbf{4 3}$ male and female undergraduates at a major northeastern university who participated in partial fulfillment of course requirements. As each of the participants evaluated four brands in each of three product categories, this yields curves representing the development of (43 participants $\times 3$ product categories $\times 4$ brands per category $=$ ) 516 attitudes toward objects. 


\section{Product Categories}

Three different product categories (CD players, clock radios, and compact refrigerators) were chosen on the basis of appropriateness for the college student participant population. The order in which the product categories were tested was counterbalanced across participants.

\section{Attributes}

Each product category was represented by a set of 20 attributes used in recent Consumer Reports reviews for evaluating brands in that category. Brands can be described in terms of a combination of positive, negative, and neutral attributes. For these purposes, a positive attribute was defined as one consisting of either (a) possession of a desirable feature (e.g., has a snooze alarm), or (b) superior performance on a particular attribute (e.g., excellent tone quality). A negative attribute was one consisting of either (a) absence of a desirable feature (e.g., does not have a snooze alarm), or (b) inferior performance on a particular attribute (e.g., poor tone quality). Neutral attributes were those on which the brand had average performance (e.g., average tone quality).

Each product category was represented by four brands: one Positive, one Negative, and two "Mixed." To enhance realism, Positive brands were not uniformly positive, and Negative brands were not uniformly negative. Positive brands were described by 18 positive attributes, two negative attributes, and zero neutral attributes; negative brands were described by 18 negative attributes, two positive attributes, and zero neutral attributes. Mixed brands contained six to eight positive attributes, six to eight negative attributes, and six to eight neutral attributes. Including both Positive and Negative brands permits determining whether attitude formation occurs in comparable fashion for both positively and negatively valenced attitudes. As negative beliefs are generally considered to be more salient than positive beliefs, negative attitudes may require fewernegative beliefs to reach asymptote. Neutral brands were included both to provide a frame of reference for the evaluations obtained for the Positive and Negative brands, and to act as "buffers" between the Positive and Negative brands.

The 20 attributes were listed in randomized order for each participant. Within each product category, the first and third brands presented for evaluation were always Mixed brands. Positive and Negative brands were inserted between Mixed brands, with the order of Positive and Negative brands being counterbalanced across categories and within respondents. Each brand was identified by a randomly chosen letter rather than a brand name.

\section{PROCEDURE}

On arrival at the test site, participants were seated at a computer workstation and told the purpose of the study was to ob- tain their evaluations of a number of different products. When they were ready to begin, participants hit a key on the keyboard and were provided with specific task instructions on screen. The first screen explained that the purpose of the study was to see how college-age consumers use computers to evaluate products. The next screen indicated they would be evaluating 12 products that were either currently available in the marketplace or now under development, and that each product would be identified by a randomly assigned letter rather than its actual brand name. Participants were told they could acquire information in any order they liked, but had to acquire at least 7 of the 20 available items of information for each brand before giving their final evaluation for that brand.

Next, participants participated in a practice task in which they evaluated a single brand of running shoes. On completion of the practice task, participants were given a written list of the 20 attributes for the first test product category, instructed to read the attribute list and to ask the experimenter for clarification of any attribute with an unclear meaning. (See Appendix for lists of attributes.) Participants then pressed any key to continue and the following appeared on screen:

Consumers differ in their opinion about products. Some things that one person likes about a product may be exactly the kinds of things another person dislikes about the same product. We'd like to find out how different features affect the way you feel about clock radios [compact refrigerators; $C D$ players]. The next screen displays 20 types of information you can use to form your evaluation.

Next, the computer listed the 20 attributes and asked the participant to indicate which he or she would like to see. Participants typed in a number, from 1 to 20 , corresponding to the information they were interested in seeing, and the requested information appeared on screen. To allow participants to consider each brand relative to other brands in the category, comparative information for other brands also appeared on screen. Participants were next asked the following:

Based on all the information you have acquired up to this point, how do you feel about Brand X? To indicate how you feel, move the cursor to the point on the "Extremely unfavorable" to "Extremely favorable" scale that best describes your feeling about Brand X. Then press the enter key.

The bottom of the screen displayed a horizontal continuum ranging from extremely unfavorable $(-100)$ to extremely favorable $(+100)$, with the cursor initially positioned at the midpoint. Participants used the arrow keys to move the cursor along the continuum, then pressed the enter key to indicate their initial evaluation. After each subsequent item of information was acquired, the cursor's opening position on the 
evaluation scale was at the point where it had last been moved by the participant.

This process continued until a participant had examined at least seven items of information for each brand. Participants who attempted to make a final evaluation before acquiring seven items of information were reminded by the computer that they needed to acquire at least seven items of information and returned them to the task. When participants indicated they were making their final evaluation, the computer program advanced to the next brand. ${ }^{1}$

\section{RESULTS}

In evaluating the 12 attitude objects, the 43 participants accessed a total of 5,093 items of information, averaging 9.87 items per evaluation. In all, they acquired 2,181 positive items, 1,897 negative items, and 1,015 neutral items. Via HOCT, each one of these 5,093 acquisitions can be tested for its impact on evaluation.

\section{Manipulation Checks}

The study included information selected to be positively, negatively, and neutrally valenced. To function as intended, the direction of attitude change should correspond to the valence of the information considered. To test this assumption, the mean attitude change caused by these three types of information was computed for each of the 20 attributes of each brand in each category, thereby providing $240(20 \times$ $4 \times 3$ ) opportunities for testing the manipulations. Of the 201 attributes that were positively or negatively valenced, 194 attitude changes were in the expected direction (39 were neutral).

An additional check was conducted by analyzing the impact of each piece of information acquired by each participant. Table 1 shows that negative, positive, and neutral information generally had negative, positive, and minimal impact on attitudes. Negative information had a positive impact on attitudes only $2.4 \%$ of the time, and positive information had a negative impact on attitudes only $2.2 \%$ of the time. In those instances where the impact was opposite to that expected, the reason seems to be a contrast effect, for example, seeing neutral information in the context of negative information, causing a positive evaluation (discussed later).

The information curves and analyses of attitude change may be artificially impacted by floor or ceiling effects with respect to the rating scales. For example, if an attitude shifts to the extreme high end of the scale early in the sequence of information acquisition, then no additional change can occur

${ }^{1}$ Prior research reveals that Higher Order Cognitive Tracing does not affect either the extent or content of information acquisition, or the dependent variable of interest (Jacoby et al., 1994).
TABLE 1

Impact of Valenced Attribute information on Attitude Formation

\begin{tabular}{lcccc}
\hline & \multicolumn{4}{c}{ Information Valence } \\
\cline { 2 - 5 } Directional Impact & & & \\
on Attitude & Negative & Neutral & Positive & Total \\
\hline Negative & 1,283 & 249 & 49 & 1,581 \\
& $(67.7 \%)$ & $(24.5 \%)$ & $(2.2 \%)$ & $(31.0 \%)$ \\
No impact & 569 & 433 & 632 & 1,634 \\
& $(30.0 \%)$ & $(42.7 \%)$ & $(29.0 \%)$ & $(32.1 \%)$ \\
Positive & 45 & 333 & 1,500 & 1,878 \\
& $(2.4 \%)$ & $(32.8 \%)$ & $(68.8 \%)$ & $(36.9 \%)$ \\
Total & 1,897 & 1,015 & 2,181 & 5,093 \\
& $(100.0 \%)$ & $(100.0 \%)$ & $(100.0 \%)$ & $(100.0 \%)$ \\
\hline
\end{tabular}

Note. Numbers in the parentheses indicate column proportions.

upward because the scale has ceiling out. We evaluated this possibility by examining the mean attitude ratings of each brand at different points in the information acquisition sequence. Floor and ceiling effects clearly were not operating. For example, at the level of the 15th piece of information accessed for the mostly positive brands, the highest mean attitude rating for a given brand was 72.4 , leaving over 25 rating scale points for additional increases. The lowest mean attitude for a mostly negative brand was 11.5 , which still left over 10 rating scale units for change.

\section{The Impact of Sequential Information}

The impact of a piece of information on a given trial, $t$, was measured by subtracting the attitude at time $t$ from the attitude at time $t-1$. The absolute value of this difference represented the overall impact of the piece of information in terms of its weighted scale value as described by Equation 2. Figure 1 presents the mean absolute change at each trial using all of the observations across products, brands, and participants. The mean values begin with Trial 2 and extend through trials for which at least 20 observations occurred (to ensure reasonably reliable indexes of change at a given trial). Impact of information was clearly largest in the earlier trials and tended to decrease (with some exceptions) up to trial 15 , where impact then tended to level off. The curve for a polynomial regression using three power terms in which the mean value was regressed onto the trial number is superimposed onto the data to suggest a smoothed function relating impact to order of information acquisition (and giving equal weights to each mean value). Statistical tests cannot be applied to this function because of error dependencies. The smoothed function is presented here as a heuristic for characterizing the impact curve.

Figure 2 presents a comparable plot, but for only products described with (almost) all positive information, Figure 3 presents the plot for products described with (almost) all negative information, and Figure 4 presents the plot for products 


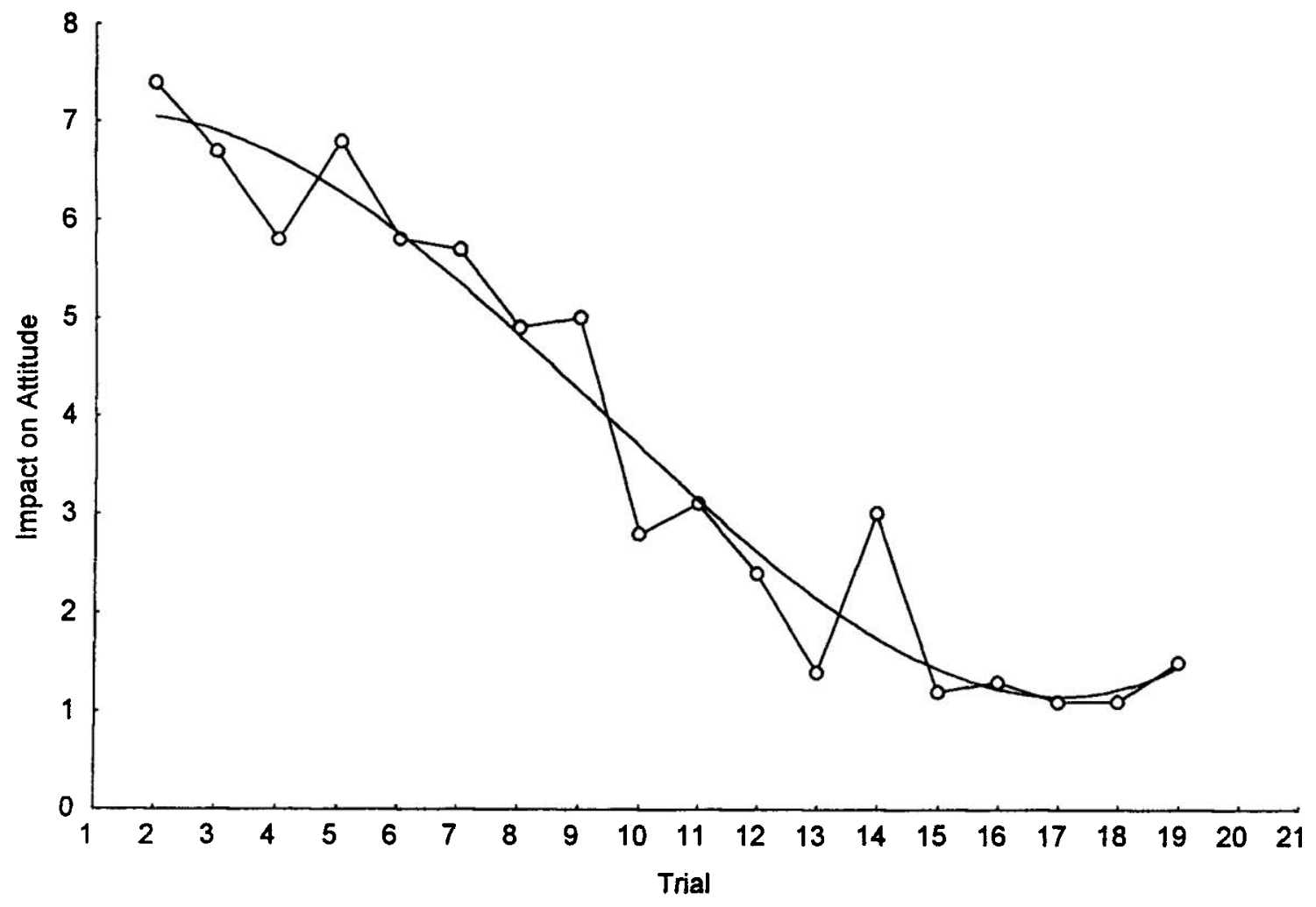

FIGURE 1 Impact of information across all 12 products.

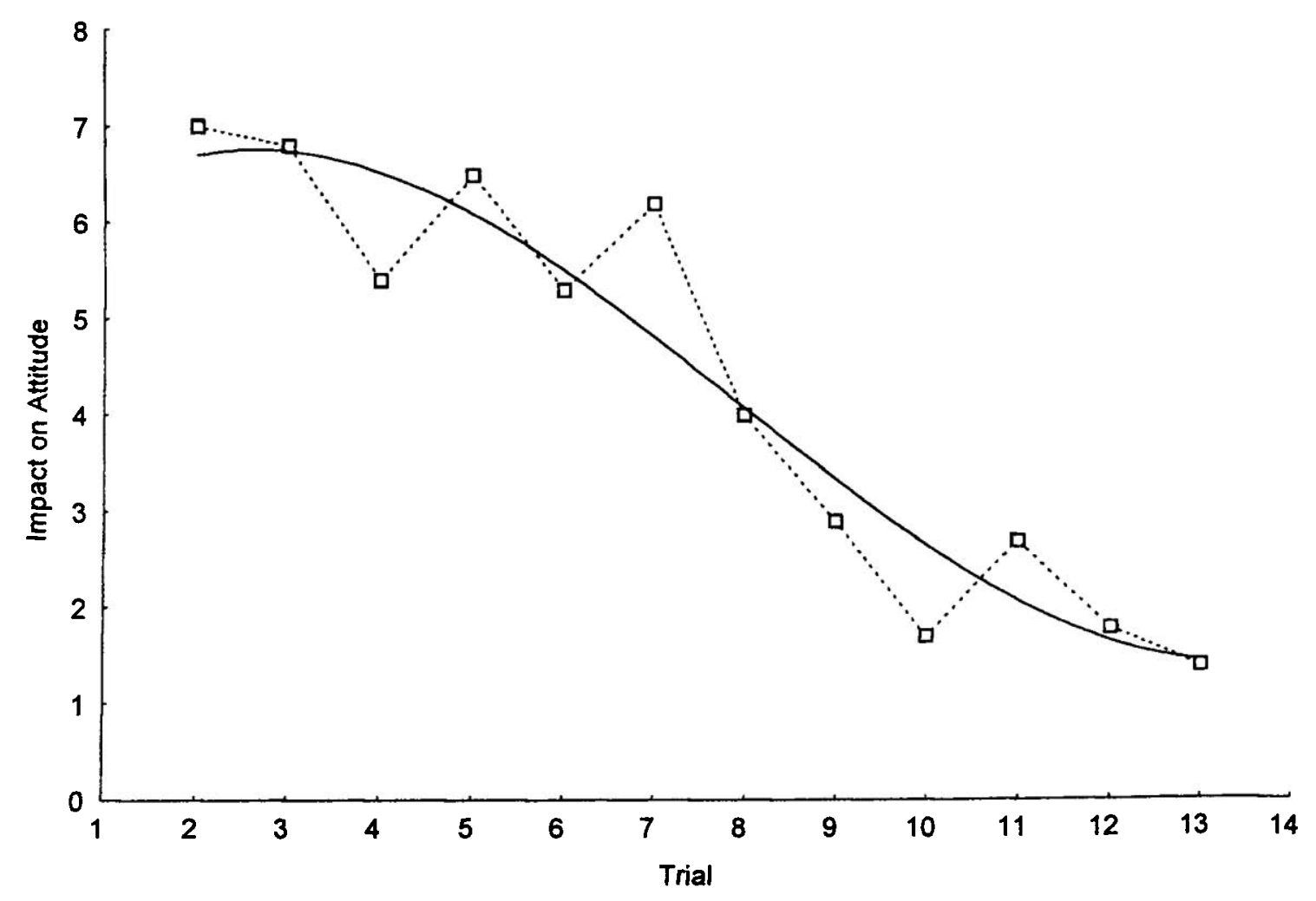

FIGURE 2 Impact of information for positive products. 


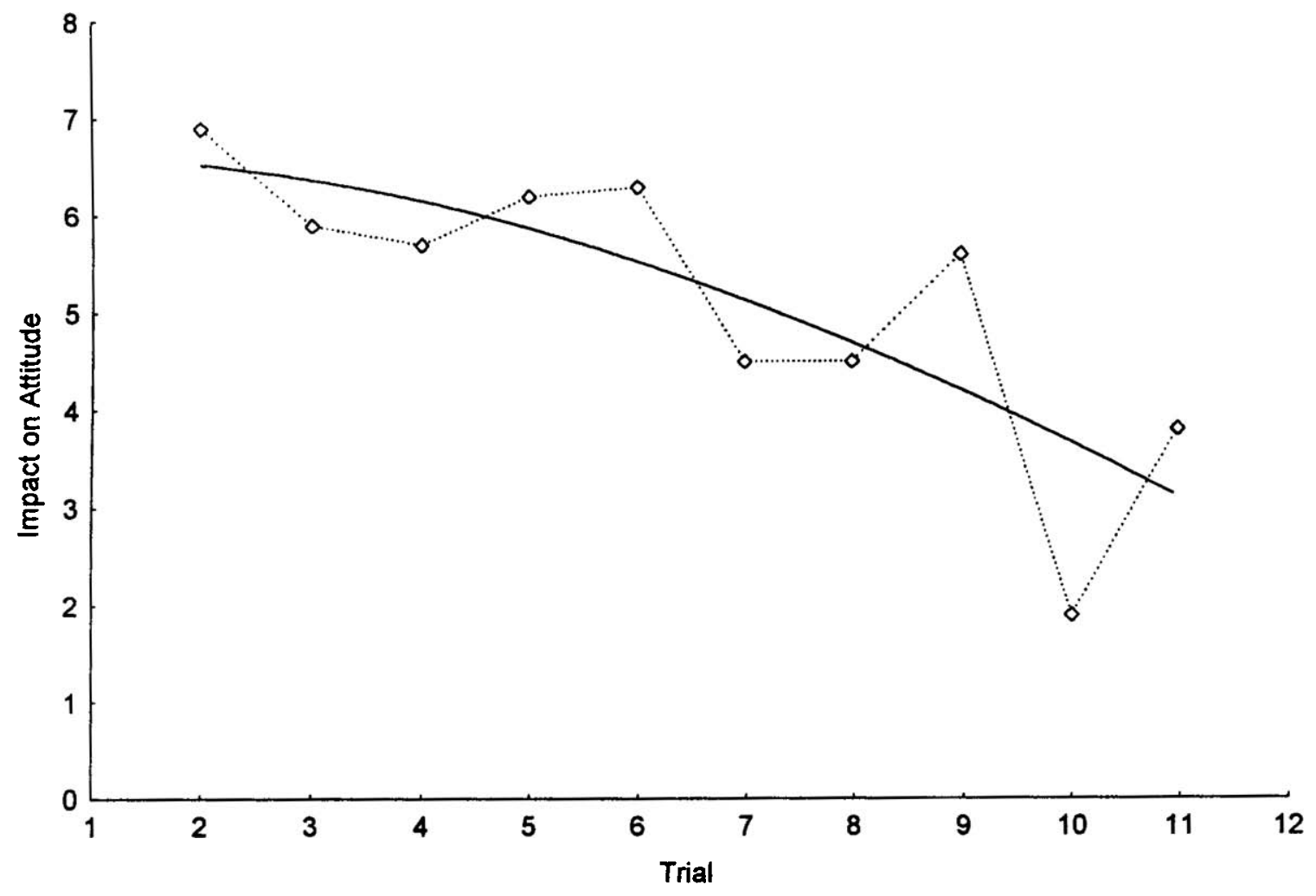

FIGURE 3 Impact of information for negative products.

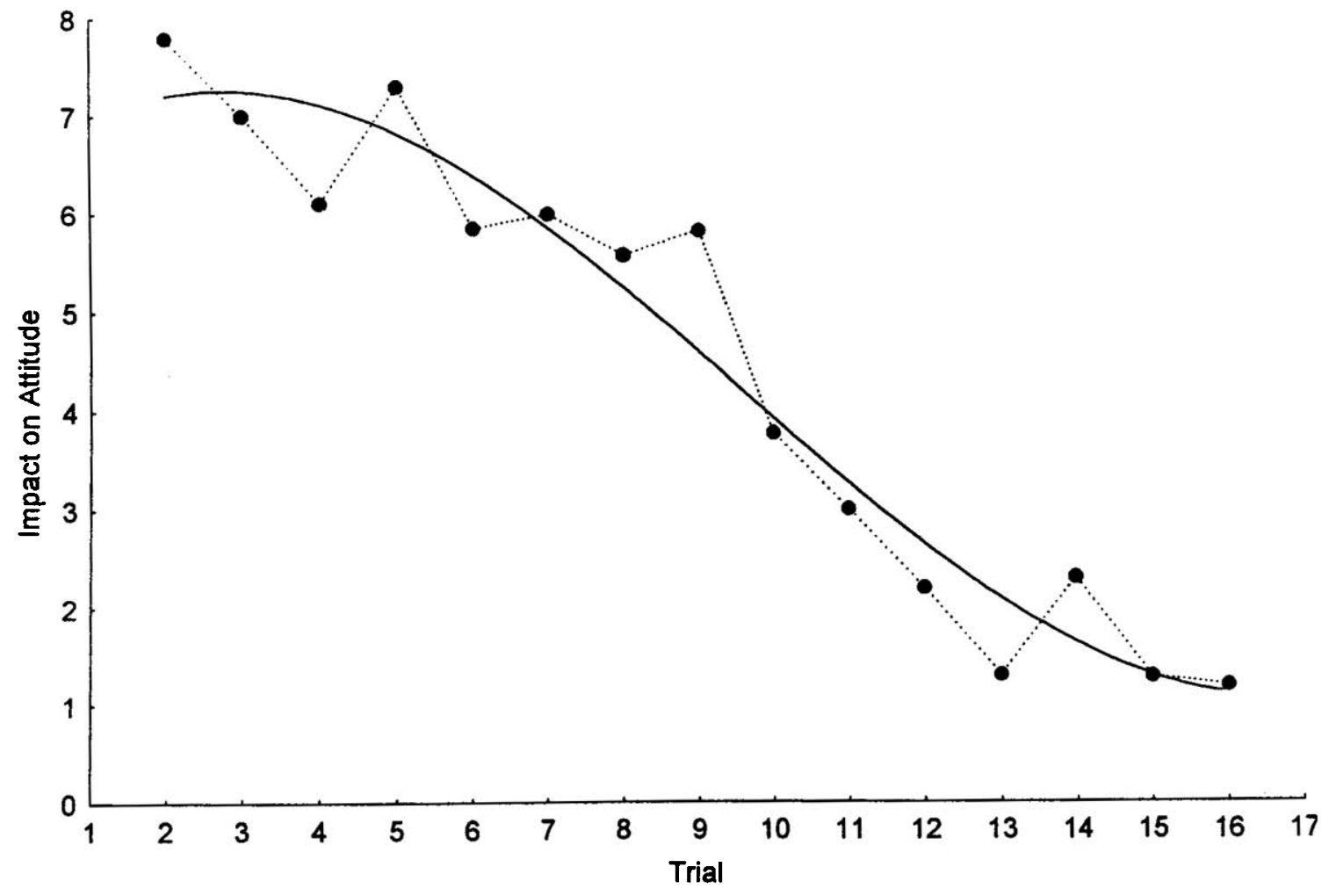

FIGURE 4 Impact of information for "mixed" products. 
characterized by both positive and negative attributes. The plot for the positive products and the mixed products are similar in form to the plot for all products and indicates a discernable drop-off of information impact. This is not true of the plot for negative products, which tends to be flatter and which exhibits less of a systematic decrease from Trials 2 through 9.

\section{Assimilation Versus Novelty Effects}

To evaluate the impact of positive versus negative information during the sequential accessing of information, we calculated the mean (signed) change in evaluation of a piece of information relative to the attitude on the preceding trial. In the case of products featured with mostly positive information, the average impact of a "novel" piece of negative information, ignoring the trial number on which it was accessed, was -9.1 as compared with a mean change of +4.2 for positive information. The standard error of the difference between these two values was 1.0 , suggesting that the almost 5 -unit absolute discrepancy between them is significant. A similar pattern of results was obtained for products featured primarily by negative attributes. When aggregated over the three products with mostly negative information, the average impact of the "novel" positive information was +9.7 as compared with -4.8 for the negative information. The standard error of the difference was also 1.0. Thus, the novel information tended to have a greater impact than the affectively redundant information, which calls into question the discounting formulation and is consistent with the novelty model.

Whereas the aforementioned tests consider the impact of positive and negative information in the context of the entire evaluation process, HOCT makes it possible to conduct more molecular tests. Thus, discounting versus novelty models may also be evaluated with respect to the case where the new information is affectively inconsistent with the piece of information accessed just prior to it. The discounting model predicts that positive information should have less of an impact when it is preceded by negative information because it is affectively inconsistent with the prior information and thereby subject to some discounting. By contrast, the novelty model predicts that positive information should have its greatest impact when accessed immediately after having ac- cessed negative information, and vice versa. The data in Table 2 are consistent with the novelty hypothesis, with the findings for positive information acquired immediately after positive, negative, or neutral information mirror-imaging those for negative information acquired immediately after positive, negative, or neutral information, respectively. These data also suggest that when acquired immediately after a negative item of information, neutral information exerts a positive impact on evaluation, but when acquired immediately after a positive item of information, neutral information exerts a negative impact on evaluation. This is consistent with an averaging integration strategy of successive information. Finally, we found that the observed novelty-contrast effect tends to decrease over the sequence of information accessing. As seen in Table 3 , when accessing sequence is divided into first-third, second-third, and third-thirds, the magnitude of average impact decreases for 15 of the 18 adjacent "thirds." The three disconfirming instances reflect only negligible increases in the magnitude of impact ( 4.2 vs. $4.8,0.3$ vs. $-0.9,13.8$ vs. 14.1; see Table 2). If we assume that, by chance, the direction of impact would be positive in half the cases and negative in half the cases, then a chi-square applied using these expected values is statistically significant $\left(\chi^{2}=8.00, p<.01\right)$.

\section{DISCUSSION}

This investigation contributes to both theory and research methodology. With respect to research methodology, this study illustrates how the Behavioral Process approach used for studying information acquisition can be extended to study the development of and changes in higher order mental processes as a function of information acquired from the external environment. This permits one to isolate and study the impact of discrete information embedded in "relatively complex messages." As noted, many of the traditional paradigms use approaches that do not permit studying changes at the level of individual pieces of information and such paradigms become difficult to implement when the information conveyed to the receiver is complex. Not only does the availability of HOCT enable one to test various process theories of attitude development-such as McGuire's (1985) construction-by-aspects formulation or Jaccard's (Jaccard \& Becker, 1985) sequential processing model — but its availability may also act as a spur

TABLE 2

Information Impact as a Function of Context Provided by Valence of Immediately Prior Information Item

\begin{tabular}{lccc}
\hline & & Current Trial (T) \\
\cline { 2 - 4 } Type Of Information In Previous Trial (T-1) & $\begin{array}{c}\text { Mean Impact of Negative } \\
\text { Information }\end{array}$ & $\begin{array}{c}\text { Mean Impact of Neutral } \\
\text { Information }\end{array}$ & $\begin{array}{c}\text { Mean Impact of Positive } \\
\text { Information }\end{array}$ \\
\hline Negative & $-2.7(1,007)$ & $4.0(244)$ & $10.8(445)$ \\
Neutral & $-7.3(257)$ & $0.6(351)$ & $6.0(305)$ \\
Positive & $-11.5(436)$ & $-3.7(308)$ & $2.4(1,224)$ \\
\hline
\end{tabular}

Note. Numbers in the parentheses indicate number of trials observed. 
to theory development and sophisticated models of attitude evolution and other social psychological phenomena (see Nowak, Lewenstein, \& Vallacher, 1994), especially those specifically postulated to operate as processes. For example, in describing the ecological view of social event perception, Berry and Misovich (1994, p. 140) wrote the following: "In addition to placing an emphasis on the dynamic nature of the stimulus information that informs perception, the ecological-event perspective conceptualizes the process of perceiving as active and dynamic." In contrast to traditional research in person perception which relies "on paradigms [involving] passive receivers" (p. 140), the ecological perspective "provide[s] researchers with some unique methodological challenges both when selecting stimuli and when assessing participants' responses to those stimuli" (p. 139). Although Berry and Misovich described various methods of stimulus presentation and response assessment, none appear as capable of assessing the dynamic development of or changes in social event perception as does HOCT. This same observation can be applied to Vallacher, Nowak, and Kaufman's (1994) use of "mouse" technology to measure the dynamics of social judgement. Other higher order phenomena that are candidates for illumination via HOCT include attribution theory, impression formation, implicit personality theory, postdecision dissonance reduction, attraction, and feedback effects, to mention but a few. Ways in which the basic procedures can be adapted to study these phenomena, including ways of constructing the available information environment to provide visual as well as alphanumeric information, are adumbrated in Jacoby, Kuss, Mazursky, and Troutman (1985) and Jacoby et al. (1987). It should also be noted that HOCT is but one of an emerging family of process methods for capturing and studying higher order mental phenomena. As described by Jacoby et al. (1994), each of these has unique advantages and disadvantages. The development and application of process methods capable of capturing dynamic mental phenomena represents an important, albeit intermediate, step. Once captured, new ways are required for describing and summarizing the data generated. Although a wide variety of approaches to developing process indexes and measures are presented and discussed elsewhere (e.g., Jacoby, Chestnut, Hoyer, \& Donahue, 1978; Jacoby, Chest- nut, Weigl, \& Fisher, 1976; Jacoby et al., 1987, pp. 151-154, 162-163), no doubt, these could be improved by being integrated with procedures suggested by Mandell and Selz (1994) and Schroeck (1994). Beyond being able to capture, describe, and analyze such processes, one would next want to model these in ways that either suggest or are consistent with theory. As Nowak, Lewenstein, and Vallacher (1994) noted

Once a pattern is identified, the next step is to try to find a system of equations that produces a similar pattern. One should also find out if this system of equations changes its pattern of behavior as a function of changes in the control parameters in a manner similar to that observed in the empirical system being modeled. If it does, then one has hit on a theoretical model that captures important aspects of the phenomenon one is trying to understand. (p. 287)

An illustration of how such an approach was applied to understanding the evolution of subjective uncertainty reduction during decision making is provided in Jacoby et al. (1994).

In terms of theory, this study yielded several interesting insights. First, as noted earlier, almost all models of attitude formation have emphasized memory-based features of the attitude formation process in which pieces of information are recalled from memory and integrated to form an attitude judgment. There has been relatively little research about online attitude formation and how attitudes emerge as information is acquired sequentially. This study found, somewhat consistent with memory-based models (Fishbein \& Ajzen, 1975), that earlier pieces of information tend to have more impact on attitudes than later information and that this holds under scenarios where individuals control the choice and order of informational dimensions that are accessed. Information impact tends to begin leveling off and to be minimal around the 10th, 11 th, or 12th piece of information acquired (see Figures 1 through 4), although this tends not to be true for the case of affectively redundant negative information (see Figure 3). The information curves showed distinct patterns for positive and negative information, with additional pieces of negative information maintaining impact for longer sequences of information acquisition. These data are consistent with the often observed negativity bias in the attitude literature (Jaccard \& Becker, 1985). These data stand in contrast to order effect analyses of

TABLE 3

Impact of Information as a Function of Affective Sign of Prior Information

\begin{tabular}{|c|c|c|c|c|c|c|c|c|c|}
\hline \multirow{3}{*}{$\begin{array}{l}\text { Type of Information In } \\
\text { Previous Trial (T-1) }\end{array}$} & \multicolumn{9}{|c|}{ Current Trial (T) } \\
\hline & \multicolumn{3}{|c|}{ Mean Impact of Negative Information } & \multicolumn{3}{|c|}{ Mean Impact of Neutral Information } & \multicolumn{3}{|c|}{ Mean Impact of Positive Information } \\
\hline & $2 \leq$ Trial $\leq 4$ & $5 \leq$ Trial $\leq 7$ & Trial $\geq 8$ & $2 \leq$ Trial $\leq 4$ & $5 \leq$ Trial $\leq 7$ & Trial $\geq 8$ & $2 \leq$ Trial $\leq 4$ & $5 \leq$ Trial $\leq 7$ & Trial $\geq 8$ \\
\hline Negative & $-3.1(379)$ & $-2.9(344)$ & $-1.9(284)$ & $4.2(69)$ & $4.8(82)$ & $3.2(93)$ & $13.8(144)$ & $14.1(159)$ & $4.2(142)$ \\
\hline Neutral & $-10.2(79)$ & $-8.2(85)$ & $-4.1(93)$ & $2.2(113)$ & $0.3(143)$ & $-0.9(95)$ & $8.0(106)$ & $6.4(92)$ & $3.8(107)$ \\
\hline Positive & $-14.0(143)$ & $-13.2(150)$ & $-7.1(143)$ & $-5.0(104)$ & $-4.4(99)$ & $-1.8(105)$ & $3.1(411)$ & $2.5(394)$ & $1.6(419)$ \\
\hline
\end{tabular}

Note. Numbers in the parentheses indicate number of trials observed. 
online processing in other domains. For example, Schlottmann and Anderson (1995) used information integration theory to study serial processing in children and found minimal order effects in the basal components of serial judgments. Johar, Jedidi, and Jacoby (1997) found evidence for recency effects, which was reduced with increasing category knowledge on the part of individuals. Hogarth and Einhorn (1992) also observed some support for recency effects. These studies, with the exception of Johar et al., have been conducted in experimental studies where individuals are forced to attend to information imposed by the experimenter. Schwarz, Strack, and Mai (1991) suggested that such scenarios produce an implied demand for attending to and revising new information. Perhaps this can account for the differences with the observed study, where individuals were free to acquire and attend to information as they saw fit. The differences with the Johar et al. study, where information acquisition was not constrained, merits further investigation.

Our research also suggested that the overall impact of a piece of information may be influenced by the extent to which it is affectively consistent with the information preceding it. Two competing models were tested, one based on a psychological model of discounting of affectively inconsistent information and the other based on a novelty effect. The results suggested that when information is affectively inconsistent with the information just prior to it, it tends to stand out, perhaps becoming more salient or diagnostic and thereby increasing its impact. These novelty effects were found to be more likely to operate early in the information acquisition sequence rather than later in the acquisition sequence. This latter finding has not been observed in prior theoretical work and could only be isolated using a methodology that examines the unfolding of attitudes as per HOCT. The results also stand in contrast to research using memory-based models in which affectively inconsistent information that is presented later in the sequence is thought to be discounted or assimilated to the earlier information.

In sum, this study has introduced a methodological strategy that represents an additional tool for consumer psychologists to study the dynamic evolution of attitudes in a dynamic information environment. Although the method has limitations, it can be used to effectively analyze a wide range of phenomena. An empirical example was provided in which HOCT was used to document information curves for different products, with distinctly different curves emerging for products that were primarily positively described, primarily negatively described, or described with a mixture of positive and negative information. Such curves have not been elaborated in the literature on order effects in attitude formation because the traditional methodological paradigms are not conducive to developing such curves. The information curves suggest that negative information retains its impact further into the information sequence than positive information. The application of HOCT also permitted us to test competing models of the impact of a piece of information that is affectively incon- sistent with the information presented just prior to it. Results supported a novelty mechanism as opposed to a discounting mechanism, with novelty effects decreasing in power as additional information is acquired.

\section{ACKNOWLEDGMENTS}

I thank Alice Eagly for her comments on an earlier version of this article.

\section{REFERENCES}

Anderson, Norman Henry. (1971). Integration theory and attitude change. Psychological Review, 78, 171-206.

Anderson, Norman Henry. (1981). Foundations of information integration theory. San Diego, CA: Academic.

Anderson, Norman Henry. (1991). Contributions to information integration theory (1, 2 and 3). Hillsdale, NJ: Lawrence Erlbaum Associates, Inc.

Berry, Diane S., \& Misovich, Stephen J. (1994). Methodological approaches to the study of social event perception. Personality and Social Psychology Bulletin, 20, 139-152.

Busemeyer, Jerome R. (1991). Intuitive statistical estimation. In Norman Anderson (Ed.), Contributions to information integration theory: Cognitive. (pp. 187-215). Hillsdale, NJ: Lawrence Erlbaum Associates, Inc.

Costanzo, Philip R. (1991, June). Socialization and mental health. Paper presented at the conference of the Society for Personality and Social Psychology, Washington, DC.

Deaux, Kay. (1993). Reconstructing social identity. Personality and Social Psychology Bulletin, 19, 4-12.

Eagly, Alice H. (1992). Uneven progress: Social psychology and the study of attitudes. Journal of Personality and Social Psychology, 63, 693-710.

Eagly, Alice H., \& Chaiken, Shelly. (1984). Cognitive theories of persuasion. In Leonard Berkowitz (Ed.), Advances in experimental social psychology (Vol. 17, pp. 267-359). San Diego, CA: Academic.

Eagly, Alice H., \& Chaiken, Shelly. (1993). The psychology of attitudes. Forth Worth, TX: Harcourt Brace.

Eiser, J. Richard. (1994). Toward a dynamic conception of attitude consistency an change. In Robin R. Vallacher \& Andrzej Nowak (Eds.), Dynamical systems in social psychology (pp. 198-218). New York: Academic.

Fishbein, Martin, \& Ajzen, Icek. (1975). Belief, attitude, intention and behavior: An introduction to theory and research. Reading, MA: Addison-Wesley.

Gergen, Kenneth J. (1973). Social psychology as history. Journal of Personality and Social Psychology, 26, 309-320.

Hastie, Reid, \& Park, Bernadette. (1986). The relationship between memory and judgment depends on whether the task is memory based or on-line. Psychological Review, 93, 258-268.

Himmelfarb, Samuel. (1973). General test of a differential weighted averaging model of impression formation. Journal of Experimental Social Psychology, 9, 379-390.

Hogarth, Robin M., \& Einhorn, Hillel J. (1992). Order effects in belief updating: The belief adjustment model. Cognitive Psychology, 24, 1-55.

Jaccard, James J., \& Becker, Michael A. (1985). Attitudes and behavior: An information integration perspective. Journal of Experimental Social Psychology, 21, 440-465.

Jaccard, James, \& Fishbein, Martin. (1975). Inferential beliefs and order effects in personality impression formation. Journal of Personality and Social Psychology, 31, 1031-1045.

Jaccard, James, Radecki, Carmen, Wilson, Tracey, \& Dittus, Patricia. (1995). Methods for identifying consequential beliefs: Implications for understanding attitude strength. In Richard Petty \& Jon Krosnick (Eds.), Attitude strength: Antecedents and consequences (pp. 236-251). Hillsdale, NJ: Lawrence Erlbaum Associates, Inc. 
Jaccard, James, Wan, Choi K., \& Wood, Gregory. (1988). Idiothetic methods for the analysis of behavioral decision making: Computer applications. In James Mancuso \& Margaret Shaw (Eds.), Cognition and personal structures: Computer access and analysis (pp. 234-256). New York: Praeger.

Jaccard, James, \& Wood, Gregory. (1986). An idiothetic analysis of consumer decision making. In David Brinberg \& Richard Lutz (Eds.), Perspectives on methodology in consumer research (pp. 67-106). New York: Springer-Verlag

Jacoby, Jacob. (1975). Consumer psychology as a social psychological sphere of action. American Psychologist, 30, 977-987.

Jacoby, Jacob. (1977). The emerging behavioral process technology in consumer decision making research. In William D. Perrault, Jr. (Ed.), $A d-$ vances in consumer research (Vol. IV; pp. 263-265).

Jacoby, Jacob, Chestnut, Robert W., Hoyer, Wayne D., \& Donahue, Michael. J. (1978). Psychometric characteristics of behavioral process data: Preliminary findings on validity and reliability. In Keith Hunt (Ed.), $A d-$ vances in consumer research (Vol. V; pp. 546-554).

Jacoby, Jacob, Chestnut, Robert W., Weigl, Karl C., \& Fisher, William. (1976). Pre-purchase information acquisition: Description of a process methodology, research paradigm and pilot investigation. In Beverlee B. Anderson (Ed.), Advances in consumer research (Vol. III; pp. 306-314).

Jacoby, Jacob, Jaccard, James J., Currim, Imran, Kuss, Alfred, Ansari, Asim, \& Troutman, Tracy. (1994). Tracing the impact of information on higher-order mental processes: The shape of uncertainty reduction. Journal of Consumer Research, 21, 291-303.

Jacoby, Jacob, Jaccard, James, Kuss, Alfred, Troutman, Tracy, \& Mazursky, David. (1987). New directions in behavioral process research. Journal of Experimental Social Psychology, 23, 146-175.

Jacoby, Jacob, Kuss, Alfred, Mazursky, David, \& Troutman, Tracy. (1985). Effectiveness of security analyst information accessing strategies: A computer interactive assessment. Computers in Human Behavior, $1_{1}$ 95-113.

Johar, Gita V., Jedidi, Kamil, \& Jacoby, Jacob. (1997). A varying parameter averaging model of on-line brand evaluations. Journal of Consumer Research, 24, 232- 247 .

Kaplan, Abraham. (1963). The conduct of inquiry. New York: Harper and Row.

Kuhn, Thomas S. (1970). The structure of scientific revolutions (2nd ed.) Chicago: University of Chicago Press.

Mandell, Arnold J., \& Selz, Karen A. (1994). The new statistical dynamics: An informal look at invariant measures of psychological time series. In Robin R. Vallacher \& Andrzej Nowak (Eds.), Dynamical systems in social psychology (pp. 55-69). New York: Academic.

McGuire, William J. (1969). The naute of attitudes and attitude change. In Gardner Lindzey and Elliot Aronson (Eds.), Handbook of social psychology (2nd ed., pp, 136-314). Reading, MA: Addison-Wesley.

McGuire, William J. (1972). Attitude change: The information processing paradigm. In Charles C. McClintlock (Ed.), Experimental social psychology (pp. 108-141). New York: Holt, Rinehart \& Winston

McGuire, William J. (1973). The yin and yang of progress in social psychology: Seven koan. Journal of Personality and Social Psychology, 26, 446-456.

McGuire, William J. (1976). Some internal psychological factors influencing consumer choice. Journal of Consumer Research, 2(4), 320-339.

McGuire, W:lliam J. (1985). Attitudes and attitude change. In Gardner Lindzey \& Elliot Aronson (Eds.), Handbook of social psychology (3rd ed., pp. 233-246). Reading, MA: Addison-Wesley.

Nowak, Andrzej, Lewenstein, Maciej, \& Vallacher, Robin R. (1994). Toward a dynamical social psychology. In Robin R. Vallacher \& Andrzej Nowak (Eds.), Dynamical systems in social psychology (pp. 279-293). New York: Academic.

Petty, Richard E., \& Cacioppo, John T. (1981). Attitudes and persuasion: Classic and contemporary approaches. Dubuque, LA: Brown.
Petty, Richard E., \& Krosnick, Jon A. (1995). Attitude strength: Antecedents and consequences. Hillsdale, $\mathrm{NJ}$ : Lawrence Erlbaum Associates, Inc.

Rosnow, Ralph L. (1981). Paradigms in transition: The methodology of social inquiry. New York: Oxford University Press.

Schlottmann, Anne, \& Anderson, Norman H. (1995). Belief revision in children: Serial judgments in social cognition and decision making domains. Journal of Experimental Psychology: Learning, Memory and Cognition, 21, 1349-1364.

Schroeck, Franklin E. Jr. (1994). New mathematical techniques for pattern recognition. In Robin R. Vallacher \& Andrzej Nowak (Eds.), Dynamical systems in social psychology (pp. 71-93). New York: Academic.

Schwarz, Norbert, Strack, Fritz, \& Mai, Hans Peter. (1991). Assimilation and contrast effects in part-whole question sequences: A conversational logic analysis. Public Opinion Quarterly, 55, 3-23.

Sloan, Lloyd R., \& Ostrom, Thomas M. (1974). Amount of information and interpersonal judgment. Journal of Personality and Social Psychology, $29,23-29$.

Torgerson, Warren S. (1958). Theory and methods of scaling. New York: Wiley.

Vallacher, Robin R., \& Nowak, Andrzej. (1994). The chaos in social psychology. In Robin R. Vallacher \& Andrzej Nowak (Eds.), Dynamical systems in social psychology (pp. 1-16). New York: Academic.

Vallacher, Robin R., Nowak, Andrzej, \& Kaufman, J. (1994). Intrinsic dynamics of social judgment. Journal of Personality and Social Psychology, 67, 20-34

Accepted by Curt Haugtvedt.

\section{APPENDIX}

\section{EXHIBIT I}

\section{ATTRIBUTE LIST FOR CD PLAYERS}

1. Ability to play damaged disks?

2. Track finding speed?

3. Resists skipping when bumped?

4. Has music sampling feature? (This is, briefly plays the introduction to each track on a disk until you press a button indicating you wish continuous play.)

5. Has random play feature? (Also called shuffle play, this feature plays all of the tracks from several disks in a random order.)

6. Displays which track on the disk is currently being played?

7. Has remote control that controls volume level?

8. Has volume fade control? (Fade control gradually lowers the volume of the music, then activates Pause. If pressed again, it gradually increases the volume.)

9. Has headphone jack?

10. Number of programmable tracks

11. Total program time displayed?

12. Warranty on parts and labor

13. Number of disks carousel holds

14. Number of disks carousel holds

15. Change disk while others play? (Allows you to change on disk while the others are still being played.)

16. Displays time left on disk? 
17. Has music peak finder? (Can locate peaks in music for purposes of setting cassette tape controls.)

18. Has delete track function?

19. Has favorite track selection?

20. Speed of fast scan

\section{EXHIBIT $\|$}

\section{ATTRIBUTE LIST FOR CLOCK RADIOS}

1. Can pick up weak FM signals?

2. Can home in one station?

3. Flutter. (Less flutter provides superior sound.)

4. Brightness display adjustable?

5. Has an earphone jack?

6. Tone quality

7. Has snooze button?

8. Also has an AM band?

9. Can set two wake-up times?

10. Indicates when alarm is set?

11. Has a separate treble control?

12. Choice of colors

13. Has a battery back-up?

14. Has automatic radio shut-off?

15. Battery strength indicator?

16. Battery strength indicator?

17. Alarm works if power fails?

18. Ease of reading display
19. Has a nap timer?

\section{EXHIBIT III}

\section{ATTRIBUTE LIST FOR COMPACT REFRIGERATORS}

1. Has automatic defrosting?

2. Estimated annual energy cost

3. Time required to freeze ice cubes

4. Has adjustable shelves?

5. Has crisper drawer to keep fruits and vegetables fresher?

6. Temperature control easy to reach?

7. Condenser coils require regular vacuuming?

8. Number of shelves

9. Freezer capacity

10. Refrigerator capacity

11. Comes with ice cube trays?

12. Has an interior light?

13. Warranty on compressor

14. Has see-through freezer door?

15. Door holds 2-liter bottles?

16. Has removable rack for eggs?

17. Choice of colors

18. Operating noise level

19. Can hold $1 / 2$ gallon containers of ice cream in freezer? 